\title{
Formation of Electronic Nematic Phase in Interacting Systems
}

\author{
Igor Khavkine ${ }^{1}$, Chung-Hou Chung ${ }^{1}$, Vadim Oganesyan ${ }^{2}$, Hae-Young Kee ${ }^{1}$ \\ 1 Department of Physics, University of Toronto, Toronto, Ontario M5S 1A7, Canada \\ 2 Department of Physics, Princeton University, Princeton, New Jersey 08544, USA
}

\begin{abstract}
We study the formation of an electronic nematic phase characterized by a broken point-group symmetry in interacting fermion systems within the weak coupling theory. As a function of interaction strength and chemical potential, the phase transition between the isotropic Fermi liquid and nematic phase is first order at zero temperature and becomes second order at a finite temperature. The transition is present for all typical, including quasi-2D, electronic dispersions on the square lattice and takes place for arbitrarily small interaction when at van Hove filling, thus suppressing the Lifshitz transition. In connection with the formation of the nematic phase, we discuss the origin of the first order transition and competition with other broken symmetry states.
\end{abstract}

PACS numbers: 71.10.Hf, 71.27.+a

\section{INTRODUCTION}

Recently, there have been reports of experimental evidence of inhomogeneous and/or anisotropic quantum ground states in strongly correlated systems 1.2 .3 .4 .5 The inhomogeneous phase - dubbed the stripe phase - was observed in high temperature cuprates via elastic neutron scattering experiments $\underline{\underline{6}}$ Theoretical studies of the inhomogeneous and anisotropic quantum ground states in connection with generic phases of a doped Mott insulator have also been carried out $\frac{7.8}{1 t}$ was suggested that as quantum fluctuation, induced by hole doping, increases, the Mott insulator turns into a smectic - stripe - phase, and a further increase of quantum fluctuation will turn a smectic to a nematic phase 7.8 The electronic smectic and nematic phases are characterized by broken translational (in one direction) and rotational symmetries, in analogy with classical liquid crystals ${ }^{9}$

The electronic nematic phase which breaks the pointgroup symmetry on a square lattice was studied in the extended Hubbard model. The instability of Fermi liquid towards possible ordered phases including the nematic - called the Pomeranchuk instability $\underline{\underline{10}}$ - were investigated, and it was shown that the nematic phase is the leading instability within some range of the parameter space 11.12 .13 Within the weak coupling theory, the effective Hamiltonian of the quadrupole-quadrupole density interaction for the nematic phase was also constructed, where the expectation value of the quadrupole density is the order parameter of the nematic phase $\underline{14}^{14}$ The study of the nematic phase and possible superconductivity in the continuum model within the weak coupling approach showed the non-Fermi liquid behavior in the single particle scattering rate $\frac{14}{4}$ and an exotic superconducting pairing symmetry 15 via the coupling to the collective modes. Possible probes of the nematic phase were also discussed, ${ }^{16.17}$

The nature of the quantum phase transition between the nematic phase and isotropic Fermi liquid as a function of chemical potential, for a particular set of parameters (interaction strength $F_{2}$ and next nearest neighbor hop- ping $t^{\prime}$ ), was recently studied numerically 18 This computation showed that the transition is generically first order. On the other hand, Ref. 19 found anisotropic non-Fermiliquid behavior at the isotropic-nematic quantum critical point - second order transition - in the presence of a lattice. Therefore it is important to investigate whether the nature of isotropic-nematic transition depends on the type of electron dispersion, the interaction strength, or the temperature.

In this paper, we investigate the behavior of the free energy and density analytically, as a function of chemical potential and interaction strength. We also extend the analysis to finite temperature and quasi-2D electron dispersion. At zero temperature, we show that the nematic transition is first order as a function of the interaction strength and chemical potential for all typical 2D electronic dispersions since they possess van Hove singularities. The transition changes to a continuous one at a finite temperature, but is not strongly affected by small dispersion in the third direction.

We also find that the transition takes place for arbitrarily small attractive interaction at the van Hove band filling. In the absence of interaction, at the van Hove filling, the Fermi surface changes topology from electronto hole-like. This transition, first studied by Lifshitz ${ }^{20.21}$, causes singularities in thermodynamic quantities such as compressibility, due to van Hove singularities, but is not accompanied by any broken symmetry. This transition was recently revisited in Ref. 22 as an example of phase transition between different quantum orders which are not classified by broken symmetry. Our findings show that the Lifshitz transition does not take place because the van Hove singularity is avoided due to a sudden change of the Fermi surface topology. This has implications for earlier studies of broken symmetry states, such as density waves. These studies 11.13.23.24.25.26 were based on the existence of the van Hove singularity, which lead to a divergence of the relevant susceptibility indicating a transition to an ordered phase. Our results suggest that these ordered phases may be preempted by the first order transition into the nematic phase.

The paper is organized as follows. We describe the 
effective model Hamiltonian for the nematic in section III The mean field analysis at zero temperature and finite temperature is given in section to other competing instabilities is discussed in section IV We also provide the summary of our findings in the last section, IV]

\section{MODEL}

\section{A. Hamiltonian}

Our choice of model Hamiltonian is largely motivated by symmetry considerations and by the philosophy of the weak-coupling BCS theory. The interaction is chosen with foresight toward the mean-field analysis and with inspiration from classical liquid crystal theory. In a liquid crystal, each rod-like molecule defines a direction in space, and the order parameter for the nematic phase is equally sensitive to their alignment as well as anti-alignment. It is a quadrupole (second order symmetric traceless) tensor built from these directions. In two dimensions, it changes sign under a rotation by $90^{\circ}$ and is invariant under a rotation by $180^{\circ}$. For an electron gas, we can construct a similar order parameter from the momenta of electrons, the quadrupole density $\hat{Q}_{i j}=\hat{p}_{i} \hat{p}_{j}-\frac{1}{2} \hat{p}^{2} \delta_{i j}$. The interaction between quadrupole densities (suitably quantized and discretized) is made attractive to favor the alignment or anti-alignment of electron momenta, i.e. formation of the nematic phase.

The precise Hamiltonian under consideration is written as

$$
H=\sum_{\mathbf{k}}\left(\varepsilon_{\mathbf{k}}-\mu\right) c_{\mathbf{k}}^{\dagger} c_{\mathbf{k}}+\sum_{\mathbf{q}} F_{2}(\mathbf{q}) \operatorname{Tr}\left[\hat{Q}^{\dagger}(\mathbf{q}) \hat{Q}(\mathbf{q})\right]
$$

where $\varepsilon_{\mathbf{k}}$ is the single-particle dispersion, $F_{2}(\mathbf{q})$ is the inter-electron interaction strength, and $\hat{Q}(\mathbf{q})$ is the the lattice analog of the quadrupole density tensor constructed in two dimensional square lattice from particle momenta given by

$$
\hat{Q}(\mathbf{q})=\frac{1}{N} \sum_{\mathbf{k}} c_{\mathbf{k}+\mathbf{q}}^{\dagger}\left(\begin{array}{cc}
\cos k_{y}-\cos k_{x} & \sin k_{x} \sin k_{y} \\
\sin k_{x} \sin k_{y} & \cos k_{x}-\cos k_{y}
\end{array}\right) c_{\mathbf{k}} .
$$

The dispersion is that of a next-nearest-neighbor tight binding model on a square lattice $\varepsilon_{\mathbf{k}}=-2 t\left(\cos k_{x}+\right.$ $\left.\cos k_{y}\right)-4 t^{\prime} \cos k_{x} \cos k_{y}$. While $F_{2}(\mathbf{q})$ is an arbitrary short ranged interaction, for example of the (two dimensional) Yukawa form,

$$
F_{2}(\mathbf{q})=-\frac{F_{2}}{2} \frac{2 \pi \kappa^{2}}{\left(1+\kappa^{2} \mathbf{q}^{2}\right)^{3 / 2}}
$$

We shall only be interested in the strength of the interaction $F_{2}$, which is assumed to be positive.

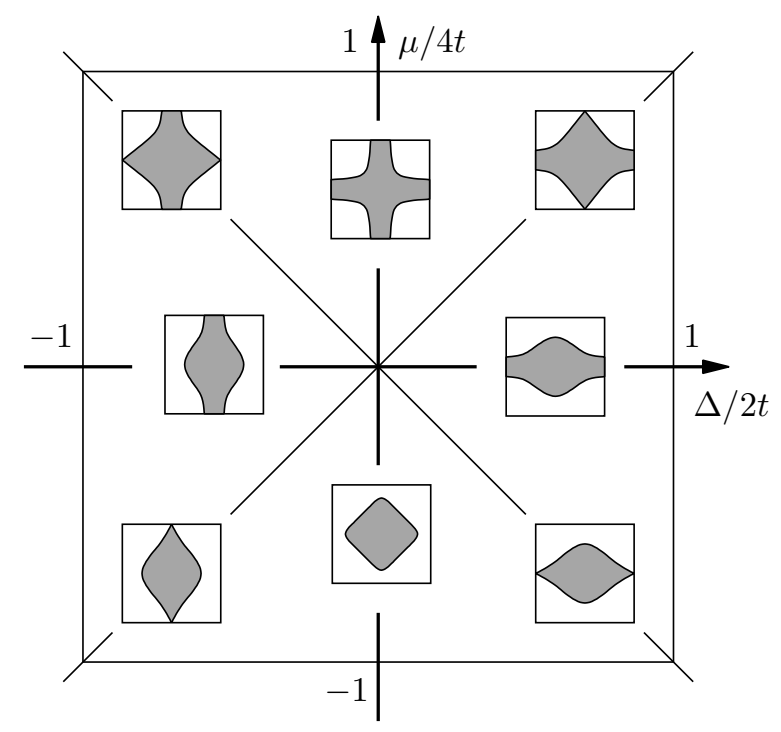

FIG. 1: Shapes of the Fermi surface (FS) for different values of $\mu$ and $\Delta$. Lines $(\mu / 2)^{2}=\Delta^{2}$ mark the van Hove singularities in the DOS which correspond to the FS touching the boundary of the Brillouin zone.

\section{MEAN-FIELD ANALYSIS}

To decouple the quartic interaction in Eq. (1), we concentrate on the $\mathbf{q}=0$ interactions (which is equivalent to letting $F_{2}(\mathbf{q}) \rightarrow-\frac{1}{2} F_{2} \delta_{\mathbf{q}, 0}$ as $\kappa \rightarrow \infty$ in Eq. (3) ) and define two order parameters,

$$
\Delta=F_{2}\left\langle\hat{Q}_{x x}(0)\right\rangle \quad \text { and } \quad \Delta^{\prime}=F_{2}\left\langle\hat{Q}_{x y}(0)\right\rangle,
$$

where the expectation value $\left\langle c_{\mathbf{k}}^{\dagger} c_{\mathbf{k}}\right\rangle$ is replaced by the Fermi distribution $n_{F}\left(\varepsilon_{\mathbf{k}}-\mu\right)$, and $\varepsilon_{\mathbf{k}}$ is the the renormalized single-particle dispersion relation

$$
\begin{aligned}
\varepsilon_{\mathbf{k}}=-2 t & \left(\cos k_{x}+\cos k_{y}\right)-4 t^{\prime} \cos k_{x} \cos k_{y} \\
& +\Delta\left(\cos k_{x}-\cos k_{y}\right)-\Delta^{\prime} \sin k_{x} \sin k_{y} .
\end{aligned}
$$

A finite $t^{\prime}$ does not qualitatively change the physics of the model. For simplicity, the value $t^{\prime}=0$ was used in the $T=0$ calculations, but a nonzero $t^{\prime}$ was used at finite temperature.

A non-zero expectation value of $\hat{Q}_{i j}$ indicates that the rotational (lattice point-group) symmetry has been broken and a preferred direction for electron momenta has been selected. In particular, a non-zero expectation value of $\hat{Q}_{x y}$ indicates that this direction is not parallel to either of the crystal axes. The profile of both the interaction and the bare dispersion favor alignment along the crystal axes, as long as the coupling constants for diagonal $\hat{Q}_{x x}$ and off-diagonal $\hat{Q}_{x y}$ elements are the same, $F_{2}^{x x}=F_{2}^{x y}$. Hence we expect $\Delta^{\prime}$ to vanish. This observation has been confirmed by numerical calculations 18

In general, unlike in the continuum mode ${ }^{14}$ with full rotational symmetry, the coupling constants $F_{2}^{x x}$ and 
$F_{2}^{x y}$ can be different. In such cases, a finite $\Delta^{\prime}$ is possible. In fact broken symmetry states with oblique alignment, such as diagonal stripes, have been discussed in Ref. 27.

\section{A. Free energy}

The resulting mean-field grand-canonical free energy density is given by

$$
F(\mu, \Delta)=\frac{1}{F_{2}} \frac{\Delta^{2}}{2}+F_{0}(\mu, \Delta)
$$

where $F_{0}$ is

$$
F_{0}(\mu, \Delta)=-\frac{1}{\beta} \int \mathrm{d} \varepsilon \mathcal{D}(\varepsilon) \ln \left(1+e^{-\beta(\varepsilon-\mu)}\right),
$$

with $\mathcal{D}(\varepsilon)$ being the density of states (DOS) of the singleparticle dispersion (5). Its exact expression is (see Appendix for details and definitions)

$$
\mathcal{D}(\varepsilon)=N_{0} \operatorname{Re}\left\{\frac{1}{\sqrt{1-\left(\frac{\varepsilon}{4 t}\right)^{2}}} K\left(1-\frac{\Delta^{2}-\left(\frac{\varepsilon}{2}\right)^{2}}{(2 t)^{2}-\left(\frac{\varepsilon}{2}\right)^{2}}\right)\right\},
$$

where $N_{0}=1 /\left(2 t \pi^{2}\right)$. For $|\varepsilon|>4 t, \mathcal{D}(\varepsilon)$ vanishes. Note the logarithmic van Hove $(\mathrm{vH})$ singularities in the DOS at $(\varepsilon / 2)^{2}=\Delta^{2}$. These singularities occur when the constant energy contour (Fermi surface) meets the boundary of the first Brillouin zone as shown in Fig. 1 Due to these remnants of the $\mathrm{vH}$ singularity of the non-interacting system, the free energy $F(\mu, \Delta)$ will exhibit non-analytic behavior at $(\mu / 2)^{2}=\Delta^{2}$. This behavior is to be contrasted with the free energy in the presence of density wave order parameters, where all singularities are smoothed. Ultimately, persistence of non-analyticities in $F(\mu, \Delta)$ leads to the first order isotropic-nematic quantum phase transition.

\section{B. Zero temperature}

First we analyze the free energy density in the limit of zero temperature $(\beta \rightarrow \infty)$. In this limit

$$
F_{0}(\mu, \Delta)=(E-\mu n)
$$

where $E$ and $n$ are the energy and particle density per unit cell.

The energy integral can be evaluated exactly, but the density integral cannot. The combined expression for the free energy (for $\Delta, \frac{\mu}{2}<2 t$, and neglecting terms independent of $\Delta$ ) is

$$
\begin{aligned}
F= & \left(\frac{1}{F_{2}}+2 N_{0}\right) \frac{\Delta^{2}}{2} \\
& +N_{0}\left[\left(\Delta+\frac{\mu}{2}\right)^{2} \ln \left|\frac{\Delta+\frac{\mu}{2}}{4}\right|+(\mu \rightarrow-\mu)\right],
\end{aligned}
$$

$$
\mu / 2 t=0
$$

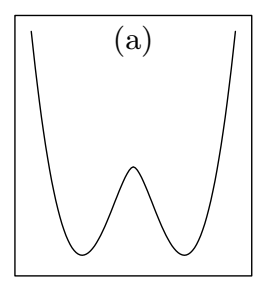

$\mu / 2 t=0.118$

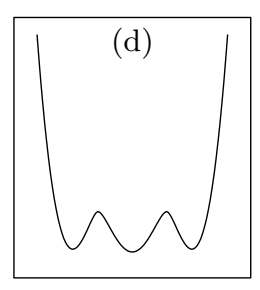

$\mu / 2 t=0.089$

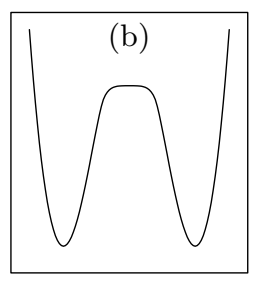

$\mu / 2 t=0.125$

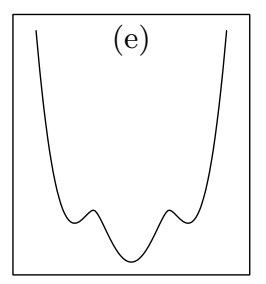

$\mu / 2 t=0.109$

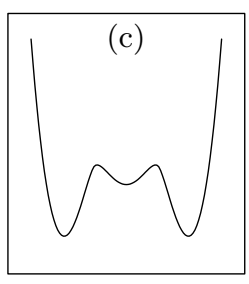

$\mu / 2 t=0.148$

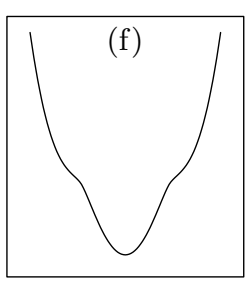

FIG. 2: Plots of free energy as a function of $\Delta$ for different values of the chemical potential $\left(F_{2} N_{0}=0.1\right)$ centered at $\Delta=0$; (b) $\mu=\mu_{*}$, (d) $\mu=\mu_{c}$ (see text).

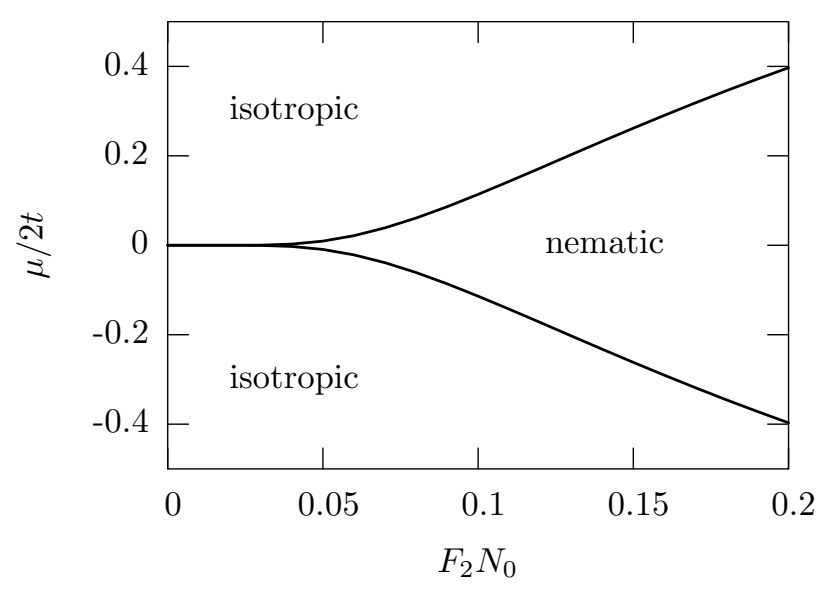

FIG. 3: Phase diagram in the $\mu-F_{2}$ parameter space. Solid curve, given by $\mu_{c}$ (12), is the line of the first order transition.

where for brevity all energy quantities are in units of 2t. Any results extracted from this expression are valid up to quadratic order in $\mu$ and $\Delta$. The details of the calculations are outlined in the Appendix.

The free energy for different chemical potentials is plotted as a function of $\Delta$ in Fig. 2. As the chemical potential decreases, the free energy develops local minima at finite $\Delta$ (the nematic phase), which then become the global minima for $|\mu|<\mu_{c}$. It is clear that the transition between isotropic and nematic phases is first order.

From Eq. (10), one can also show that $\Delta=0$ is always an extremum of the free energy. However, no local minimum of the free energy can be located in the region 


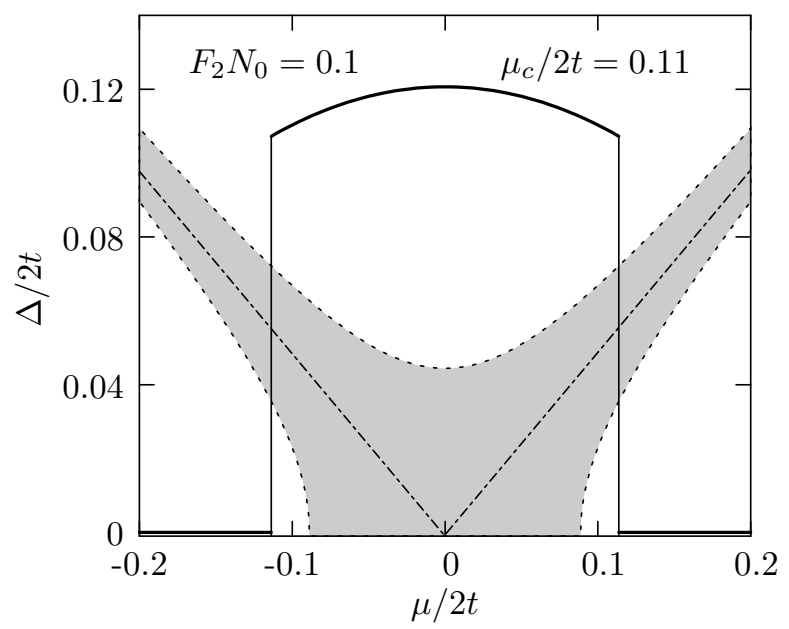

FIG. 4: Equilibrium value of the order parameter $\Delta$. The shaded region indicates negative curvature of the free energy. Lines $\Delta^{2}=\left(\frac{\mu}{2}\right)^{2}$ are marked by dot-dashed lines.

$\left|\left(\frac{\mu}{2}\right)^{2}-\Delta^{2}\right|<\left(\frac{\mu_{*}}{2}\right)^{2}$, where

$$
\mu_{*} / 2 t=1.08 e^{-\frac{1}{4 F_{2} N_{0}}},
$$

this is the limit of metastability of the isotropic phase $(\Delta=0)$ which becomes unstable for $|\mu|<\mu_{*}$. Hence, $\Delta$ must have a finite equilibrium value in this region (nematic phase). The phase transition actually takes place slightly outside this region at $|\mu|=\mu_{c}$,

$$
\mu_{c} / 2 t=1.39 e^{-\frac{1}{4 F_{2} N_{0}}} .
$$

We stress again that $\mu_{c}>\mu_{*}$, which indicates that the nematic transition takes precedence over the Pomeranchuk instability (divergence of susceptibility). In Fig. 3 we show the line of the first order phase transition in the parameter space of interaction strength, $F_{2}$, and chemical potential.

The nontrivial local minima are located at

$$
\pm \Delta / 2 t=1.47 e^{-\frac{1}{4 F_{2} N_{0}}}-1.36 e^{\frac{1}{4 F_{2} N_{0}}}\left(\frac{\mu}{2 t}\right)^{2} .
$$

Eq. 13 is valid for $|\mu|<\mu_{c}$. The Fig. [4 shows the behavior of the order parameter. The order parameter jump and the width of the nematic window decrease exponentially as the coupling strength $F_{2}$ goes to 0 . The unstable

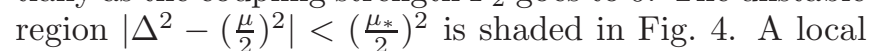
minimum of the free energy can only be found outside this area, which explains the discontinuous character of the phase transition, since the equilibrium value of the order parameter must jump to avoid it. The dot-dashed lines, $\left(\frac{\mu}{2}\right)^{2}=\Delta^{2}$, are where the change in topology of the Fermi surface takes place, the Lifshitz transition. As shown in Fig. [4 these lines are embedded in the unstable region. Therefore, our results indicate that, in the presence of interaction, the Lifshitz transition is not realized due to the formation of the nematic phase.

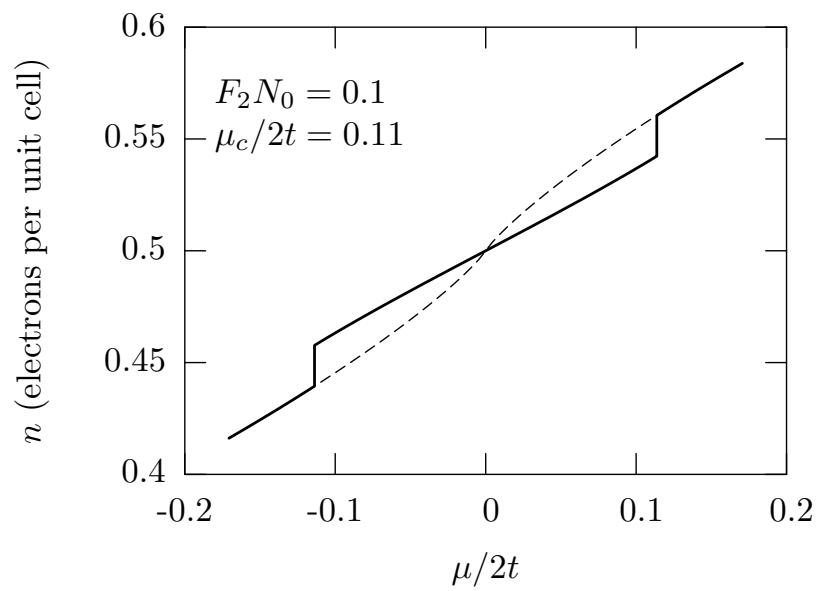

FIG. 5: The electron density per unit cell. The jump in the density is a signature of a first order phase transition.

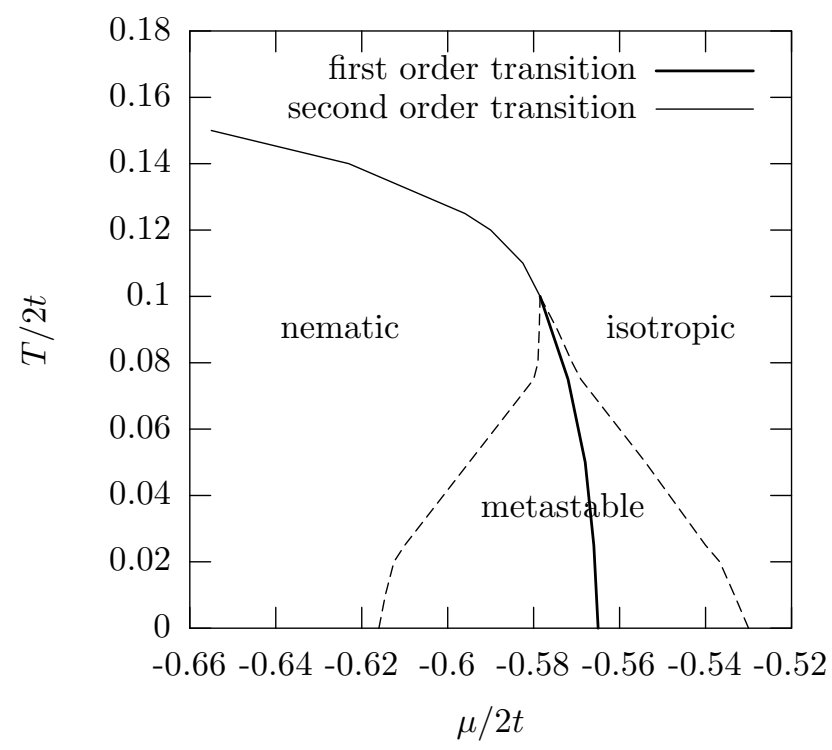

FIG. 6: The finite temperature phase diagram for the isotropic-nematic transition. The first order transition persists at low temperatures. However, the metastable region gets smaller and smaller with increasing temperature. Finally, around $T / 2 t=0.1$, the phase transition becomes continuous.

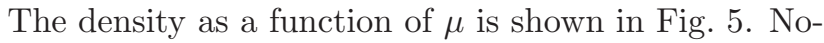
tice that the dotted line is the density in the absence of nematic order. Its derivative at $\mu=0$ is singular, which signals the Lifshitz transition. However, once the nematic order sets in, the density at half filling becomes smooth, but shows a discontinuity at $\mu_{c}$. 


\section{Finite temperature}

To investigate the robustness of the first order isotropic-nematic transition, we study the transition at finite temperature and with a finite inter-plane hopping term $t_{z}$. These calculations are performed numerically using the same technique as in Ref. 18. The first order transition is robust against a small $t_{z}=0.1 t$ term, but changes to a second order one at a finite temperature.

The phase diagram for finite temperatures is shown in Fig. 6] for a fixed $F_{2} N_{0}=0.11$ and a finite next nearest neighbor hopping $t^{\prime}\left(t^{\prime}=-0.4 t\right)$. The full width of the nematic window is from $\mu / 2 t=-0.94$ to -0.57 .18 It is worth noting that the first order transition does not alter qualitatively with a finite $t^{\prime}$.

A negative (positive) $t^{\prime}$ shifts the window of the nematic phase to the hole- (electron-) doped side. At low temperatures the transition is still first order surrounded by a metastable region (which indicates the presence of unstable local minima). However, at about $T / 2 t=0.1$, the metastable region disappears and the transition becomes continuous.

The discontinuity in the isotropic-nematic transition at zero temperature can be traced to the presence of the lattice, which dictates the form of the dispersion relation and the presence of the van Hove singularity, and the sharpness of Fermi distribution. With increasing temperature, the thermal fluctuation will smear the sharpness of the Fermi distribution function, which results in a smaller jump in the order parameter and finally in a continuous transition. This expectation is confirmed by the numerics presented here.

\section{DISCUSSION AND SUMMARY}

There has been a number of one-loop renormalization group studies for the Hubbard model taking into account the Fermi surface only at the saddle points, $( \pm \pi, 0)$ and $(0, \pm \pi)$, namely the two patch model at van Hove filling. These studies showed that there are antiferromagnetic and $d$-wave pairing instabilities in Hubbard model ${ }^{23,24.28}$ Recently the two patch model was revisited, and truncation of the Fermi surface near the saddle points was suggested ${ }^{25}$

On the other hand, the instability of the Fermi liquid towards the formation of the nematic phasePomeranchuk instability - with other competing orders were also recently investigated using different methods in the extended Hubbard mode111,12,13,26 and the $t-J$ model. ${ }^{29}$ In Ref. 11, the authors noticed that the nematic instability is driven by the attractive (repulsive) interaction between electrons in the same (different) patches via forward scatterings in the Hubbard model. This finding is consistent with our effective Hamiltonian, where $-F_{2}\left(\cos k_{x}-\cos k_{y}\right)\left(\cos k_{x}^{\prime}-\cos k_{y}^{\prime}\right)$ suggests an attractive interaction between electrons near $( \pm \pi, 0)$ and $( \pm \pi, 0)$, and a repulsive one between $( \pm \pi, 0)$ and $(0, \pm \pi)$. While the understanding of the effective interaction for the nematic phase from the microscopic Hamiltonian is still missing, it suggests that the effective nematic interaction is hidden in the extended Hubbard model.

Our discovery of a strong tendency toward the nematic phase near van Hove filling suggests that the two patch model should be revisited. Since the nematic order occurs for extremely small interaction near van Hove filling, the existence of saddle points itself should be addressed. Since the Fermi surface topology "suddenly" changes from closed to open as indicated in our result, we speculate that the instability towards competing orders such as antiferromagnetic and charge density wave (which are sensitive to the topology of the Fermi surface and enhanced by van Hove singularity) would be suppressed by the formation of the nematic phase.

In summary, we have investigated a model Hamiltonian exhibiting the nematic phase. At zero temperature the isotropic-nematic transition takes place for arbitrarily small coupling at van Hove band filling. Away from the van Hove filling, a finite minimum interaction is required to stabilize the nematic phase. The phase transition is first order as a function of interaction strength (chemical potential) for a fixed chemical potential (interaction strength) as shown in Fig. 3 The strong tendency toward the nematic phase for an arbitrary small interaction at van Hove filling suggests that the Lifshitz transition is suppressed in the presence of interactions. At a finite temperature the transition becomes second order, while it remains first order for a quasi-2D dispersion.

\section{Acknowledgments}

This work was supported by NSERC of Canada (IK, CHC, HYK), Canada Research Chair (HYK), Canadian Institute for Advanced Research (HYK), Alfred P. Sloan Research Fellowship (HYK), and Emerging Material Knowledge program funded by Materials and Manufacturing Ontario (HYK). VO thanks W. Metzner and D. Huse for discussions. VO is supported by grants from NSF (DMR 99-78074 and DMR 02-13706), and from the David and Lucille Packard foundation.

\section{APPENDIX A: DERIVATION OF FREE ENERGY}

The Density of states is defined and evaluated as

$$
\begin{aligned}
& \mathcal{D}(\varepsilon)=\frac{1}{N} \sum_{\mathbf{k}} \delta\left(\varepsilon-\varepsilon_{k}\right) \\
& =N_{0} \operatorname{Re}\left\{\frac{1}{\sqrt{1-\left(\frac{\varepsilon}{4 t}\right)^{2}}} K\left(1-\frac{\Delta^{2}-\left(\frac{\varepsilon}{2}\right)^{2}}{(2 t)^{2}-\left(\frac{\varepsilon}{2}\right)^{2}}\right)\right\},
\end{aligned}
$$

where $K(m)$ is the Complete Elliptic Integral of the First Kind, ${ }^{30}$ and $N_{0}=1 /\left(2 \pi^{2} t\right)$. The function $K(m)$ has a 
logarithmic singularity at $m=1$. At zero temperature, the free energy density is (cf. Eq. (6))

$$
F=\frac{1}{F_{2}} \frac{\Delta^{2}}{2}+(E-\mu n) .
$$

Here $E$ is the energy density

$$
\begin{gathered}
E=\int_{-4 t}^{\mu} \mathrm{d} \varepsilon \varepsilon \mathcal{D}(\varepsilon) \\
=-(4 t)^{2} N_{0} \operatorname{Re}\left\{\sqrt{1-\left(\frac{\mu}{4 t}\right)^{2}} E\left(1-\frac{\Delta^{2}-\left(\frac{\mu}{2}\right)^{2}}{(2 t)^{2}-\left(\frac{\mu}{2}\right)^{2}}\right)\right\},
\end{gathered}
$$

where $E(m)$ is the Complete Elliptic Integral of the Second Kind ${ }^{30}$ And $n$ is the electron density

$$
\begin{aligned}
& n=\int_{-4 t}^{\mu} \mathrm{d} \varepsilon \mathcal{D}(\varepsilon) \approx \frac{1}{2}+\left(2 t N_{0}\right) \mu \\
& +\left(2 t N_{0}\right)\left[\left(\Delta-\frac{\mu}{2}\right) \ln \frac{1}{4}\left|\Delta-\frac{\mu}{2}\right|-(\mu \rightarrow-\mu)\right] .
\end{aligned}
$$

This expression is a leading order expansion in $\left(\Delta \pm \frac{\mu}{2}\right)$. Here, for brevity, $\mu$ and $\Delta$ are in units of $2 t$.

Expanding the energy density to the same order, the combined free energy (neglecting terms independent of $\Delta$ ) is given in Eq. (10). Equating to zero the first derivative of the free energy with respect to $\Delta$ gives an equation for its local extrema. Nontrivial minima are easily found at $\mu=0$. In Eq. (13) location of these minima is given to quadratic order in $\mu$. Using this expression we can find the chemical potential $\mu_{c}$ (Eq. (12) at which they become global minima. Equating to zero the second derivative of the free energy with respect to $\Delta$, we find the chemical potential $\mu_{*}$ (Eq. (11)) at which the extremum at $\Delta=0$ changes from a local minimum to a maximum.
1 S. Mori, C. H. Chen, and S. W. Cheong, Nature 392, 473 (1998).

2 M. P. Lilly, K. B. Cooper, J. P. Eisenstein, L. N. Pfeiffer, and K. W. West, Phys. Rev. Lett. 82, 394 (1999), cond-mat/9903196.

3 R. R. Du, D. C. Tsui, H. L. Stormer, K. B. Cooper, L. N. Pfeiffer, K. W. Baldwin, and K. W. West, Solid State Comm. 109, 389 (1999), cond-mat/9812025.

${ }^{4}$ K. B. Cooper, M. P. Lilly, J. P. Eisenstein, L. N. Pfeiffer, and K. W. West, Phys. Rev. B 65, 241313 (2002), cond-mat/0203174.

5 Y. Ando, K. Segawa, S. Komiya, and A. N. Lavrov, Phys. Rev. Lett. 88, 137005 (2002), cond-mat/0108053.

6 J. Tranquada, B. J. Sternlieb, J. D. Axe, Y. Nakamura, and S. Uchida, Nature 375, 561 (1995).

7 S. A. Kivelson, E. Fradkin, and V. J. Emery, Nature 393, 550 (1998), cond-mat/9707327.

8 E. Fradkin and S. A. Kivelson, Phys. Rev. B 59, 8065 (1999), cond-mat/9810151.

9 P. de Gennes, Physics of Liquid Crystals (Oxford University Press, 1987).

10 I. J. Pomeranchuk, Sov. Phys.-JETP 8, 361 (1958).

11 C. J. Halboth and W. Metzner, Phys. Rev. Lett. 85, 5162 (2000), cond-mat/0003349.

12 V. Hankevych and F. Wegner, Phys. Rev. B 31, 333 (2003), cond-mat/0207612.

13 A. P. Kampf and A. A. Katanin, Phys. Rev. B 67, 125104 (2003), cond-mat/0212190.

14 V. Oganesyan, S. Kivelson, and E. Fradkin, Phys. Rev. B 64, 195109 (2001), cond-mat/0102093.

15 Y. B. Kim and H.-Y. Kee, J. Phys. Cond. Matt. 16, 3139
(2004), cond-mat/0204037.

16 H.-Y. Kee, Phys. Rev. B 67, 073105 (2003), cond-mat/0208524.

17 S. A. Kivelson, E. Fradkin, V. Oganesyan, I. P. Bindloss, J. M. Tranquada, A. Kapitulnik, and C. Howald, Rev. Mod. Phys. 75, 1201 (2003), cond-mat/0210683.

18 H.-Y. Kee, E. H. Kim, and C.-H. Chung, Phys. Rev. B 68, 245109 (2003), cond-mat/0304467.

19 W. Metzner, D. Rohe, and S. Andergassen, Phys. Rev. Lett. 91, 066402 (2003), cond-mat/0303154.

20 I. M. Lifshitz, Sov. Phys.-JETP 11, 1130 (1960).

21 I. M. Lifshitz, JETP 38, 1569 (1960).

22 X.-G. Wen, Phys. Lett. A 300, 175 (2002), cond-mat/0110397.

${ }^{23}$ H. J. Schulz, Europhys. Lett. 4, 609 (1987).

24 P. Lederer, G. Montambaux, and D. Poilblanc, J. Phys. (Paris) 48, 1613 (1987).

25 N. Furukawa, T. M. Rice, and M. Salmhofer, Phys. Rev. Lett. 81, 3195 (1998), cond-mat/9806159.

26 C. Honerkamp, M. Salmhofer, and T. M. Rice, Eur. Phys. J. B 27, 127 (2002), cond-mat/0204063.

27 M. Fujita, K. Yamada, H. Hiraka, S. H. Lee, S. Wakimoto, and G. Shirane, Phys. Rev. B 65, 064505 (2002), cond-mat/0101320.

28 I. E. Dzyaloshinskii, Sov. Phys.-JETP 66, 848 (1987).

29 H. Yamase and H. Kohno, J. Phys. Soc. Jpn. 69, 2151 (2000).

30 M. Abramowitz and I. S. eds., Handbook of Mathematical Functions (National Bureau of Standards, 1964), Chapter 17 (Elliptic Integrals), 10th printing, 1972. 